\title{
Motion onset really does capture attention
}

\author{
Kendra C. Smith ${ }^{1}$ (iD $\cdot$ Richard A. Abrams ${ }^{1}$
}

Published online: 3 July 2018

(C) The Psychonomic Society, Inc. 2018

\begin{abstract}
Several properties of visual stimuli have been shown to capture attention, one of which is the onset of motion. However, whether motion onset truly captures attention has been debated. It has been argued that motion onset only captured attention in previous studies because properties of the animated motion used in those experiments caused it to be "jerky" (i.e., there were gaps between successive images during animated motion). The present study sought to determine whether natural motion onset captures attention. Additionally, the present study further examined the circumstances under which animated motion onset, the only type of motion onset that can be produced on a computer display, does and does not capture attention. In Experiment 1, participants identified target letters in search arrays containing distinct animated motion types, either accompanied or unaccompanied by a new object. Animated motion onset captured attention, but not when the motion onset was accompanied by a new object, indicating that prior failures to replicate capture by animated motion onset were limited because a new object had always been included in the display. Experiment 2 employed natural motion rather than animated motion and found that participants were fastest at identifying motion-onset targets compared to other target types. These results provide further support for the claim that motion onset captures attention.
\end{abstract}

Keywords Attention $\cdot$ Attentional capture $\cdot$ Motion onset $\cdot$ Motion perception

\section{Introduction}

William James (1890) wrote "Everyone knows what attention is. It is the taking possession by the mind in clear and vivid form, of one out of what seem several simultaneously possible objects or trains of thought." Attending to something makes it the subject of our conscious experience. But how do we select the objects to which we attend?

Attentional capture is guided by the interaction of goals (top-down processes), salience (bottom-up processes), and selection history (Awh, Beloposky, \& Theeuwues, 2012). Topdown processes of attentional capture are goal-driven. For example, attempting to find a friend in a crowd may be a goal used to guide the allocation of attention. If one knows that the friend is wearing an orange shirt, orange objects in the visual field will be attended. Alternatively, bottom-up processes are thought to be automatic in nature. For example, a lone orange

Kendra C. Smith

kendrasmith@wustl.edu

1 Department of Psychological and Brain Sciences, Washington University in St. Louis, St. Louis, MO, USA in a bushel of apples may automatically attract attention because it is unique.

In experiments examining attentional capture, a certain property may be said to capture attention if an advantage is seen for the property even when it is no more likely to signal a target than any other property (e.g., Abrams \& Christ, 2003; Sunny \& von Mühlenen, 2011). Because there is no incentive to allocate attention towards a specific property in this type of experimental design, attentional capture is said to be bottomup.

Abrams and Christ (2003) found that one property of objects that captures attention in a bottom-up manner is the onset of motion. They found that a target that underwent a motion onset was identified significantly faster than other target types in a visual search task.

They compared attentional capture by continuously moving objects, stationary objects that began moving (motion-onset objects), static objects, and objects that were initially moving but then stopped moving (motion-offset objects). The findings indicated that motion onset captures attention automatically, in a bottom-up manner.

However, the finding that motion onset captures attention has been debated. Sunny and von Mühlenen (2011) argued that Abrams and Christ found attentional capture by motion 
onset because the animated motion they used was "jerky." Motion on a computer monitor differs from real, natural motion. On a computer monitor, a moving display consists of a sequence of still frames updated at a rapid rate, often 60-120 frames $/ \mathrm{s}(60-120 \mathrm{~Hz})$, to create an animated scene. Animated motion may appear smooth because of mechanisms of the visual system, such as iconic memory (e.g., Shioiri \& Cavanagh, 1992), which allow successive frames to be joined together to produce an illusion of movement. Frames are produced so rapidly that successive static images make the motion appear smooth and continuous (an example of apparent motion) even though the animated objects that appear to be moving are simply disappearing in one location and reappearing in another. Thus, motion on a computer monitor is simulated rather than natural.

In the experiments by Abrams and Christ, the refresh rate of the animated motion was $15 \mathrm{~Hz}$, which Sunny and von Mühlenen called "jerky." Since the properties inherent to animated motion at a slow refresh rate, such as the larger distance between stimuli as they are redrawn (given a constant speed), may have captured attention, Sunny and von Mühlenen investigated whether changing the display refresh rate would change the effect of attentional capture by motion onset. They found that a motion onset object was identified as quickly as a new object at low refresh rates but no faster than a static object at high refresh rates. Thus, they concluded that smooth motion onset does not capture attention, whereas jerky motion onset does. Sunny and von Mühlenen (2011) took their results as evidence that motion onset per se does not capture attention; rather, it is the offset of an object in one location and its onset in another that captures attention. In a subsequent set of experiments, Sunny and von Mühlenen replicated their earlier findings (Sunny \& von Mühlenen, 2014).

\section{Attentional capture by new objects}

Importantly, there was a critical aspect of the experiments by Sunny and von Mühlenen $(2011,2014)$ that limited the conclusions that are possible about motion onset. In those experiments, a new object appeared in the display on each trial coincident with the onset of motion. Because abrupt onsets of objects are known to capture attention (e.g., Christ \& Abrams, 2006; Enns, Austen, Di Lollo, Rauschenberger, \& Yantis, 2001; Schrij, Owens, \& Theeuwes, 2008, Yantis \& Johnson, 1990; Yantis \& Jonides, 1984), the inclusion of a new object on each trial may have obscured any effects of capture by motion onset. Indeed, it is known that abrupt onsets are prioritized; for example, Enns et al. (2001) showed that new objects were prioritized with respect to old objects that underwent a large luminance change. Additionally, in experiments by Christ and Abrams (2006), participants identified target letters at cued locations sometimes accompanied by the onset of new distractor letters. Even when participants knew with certainty where the target would appear, their attention was captured by new objects in the display such that reaction times for targets were slower when there was an abrupt onset object in the display compared to when there was not, indicating that new objects capture attention automatically and cannot be ignored. Therefore, the new object included in the experiments by Sunny and von Mühlenen $(2011,2014)$ most likely captured attention and altered the pattern of capture by motion onset.

Supporting these conjectures, there has been research showing that a new object can suppress the effect of capture by motion onset. Christ and Abrams (2008) examined cooccurring new objects and motion onset. In their experiment, two static placeholders were initially displayed. Then, those placeholders changed into letters and, simultaneously, two new letters appeared, one of which was moving. This yielded a static object, a preexisting motion onset object, a new moving object, and a new static object. Christ and Abrams found that motion onset captured attention in the presence of static objects but that new objects, whether static or moving, captured attention in the presence of preexisting objects that underwent a motion onset or preexisting static objects. Given these findings, the experiments by Sunny and von Mühlenen $(2011,2014)$ were weak tests of capture by motion onset because they used a task in which a motion onset and a new object occurred simultaneously. Because the method used to study attentional capture in Sunny and von Mühlenen $(2011,2014)$ was flawed, it is important to determine whether motion onset truly captures attention.

\section{Present study}

The present study sought first to determine if including a new object in the display alters the effect of attentional capture by (animated) motion onset (Experiment 1). If motion onset fails to capture attention when a new object is included in the display but captures attention when a new object is not included, those results would indicate that the method of Sunny and von Mühlenen (2011) did not adequately test attentional capture by motion onset. Second, the present study sought to determine if natural, analog motion onset captures attention (Experiment 2). If so, it can be concluded that real motion onset does indeed capture attention.

\section{Experiment 1}

Experiment 1 conceptually replicated the experiments by Abrams and Christ (2003) and Sunny and von Mühlenen (2011). In this experiment, some of the trials included a new object, as in the experiments by Sunny and von Mühlenen (2011), and some of the trials did not include a new object, as in the experiments by Abrams and Christ (2003). Including 
both trial types in the same experiment allowed comparison of identical animated motion across trial types. Importantly, if motion onset captures attention when there is no new object but fails to capture attention in the presence of a new object, the results would indicate that the experiments in Sunny and von Mühlenen (2011) were not good tests of attentional capture by motion onset (a conclusion that is further supported by the results of Christ \& Abrams, 2008). Because the motion onsets in this experiment were animated, simulated motion onsets (i.e., rendered on a computer display), the results cannot show whether true motion onset captures attention; this experiment only sought to determine whether including a new object alters the pattern of attentional capture by animated motion onset.

\section{Method}

Participants Twelve participants recruited from the subject pool at Washington University in St. Louis participated for course credit. This sample size is typical of experiments examining attentional capture by motion onset, which have included between 10 and 14 participants (Abrams \& Christ, 2003; Sunny \& von Mühlenen, 2011).

Procedure There were two trial types that were intermixed throughout the experiment. One trial type was a replication of the Abrams and Christ (2003) method, which will be referred to as No New Object trials. The sequence of events on trials of this type is shown in Fig. 1. In these trials, four figureeight placeholders $\left(2^{\circ}\right.$ high, $1^{\circ}$ wide $)$ were distributed on the screen around a central fixation dot. The placeholders each traveled in a circular path (radius $1^{\circ}$ ) centered on one of the corners of an $11^{\circ}$ square. The starting location of each placeholder was randomly selected to be a point along the circular path it made during rotation. The display refreshed at a rate of $42.5 \mathrm{~Hz}-$ i.e., successive renderings of moving placeholders were redrawn at that rate. At the beginning of each trial, when the placeholders initially appeared, two were moving at the rate of $348 \%$ s (one clockwise and one counterclockwise), and two were stationary. The motion continued for 3,200 ms, at which time a display transition occurred. At the time of display transition, (1) the placeholders all changed to letters, (2) one of the initially moving placeholders stopped moving, and (3) one of the initially stationary placeholders started moving (see Fig. 1). The transition produced four object types: a motion onset object (placeholder was initially stationary but changed to a letter and began rotating at the display transition), a motion offset object (placeholder was initially moving but stopped moving at the display transition), a continuous motion object (placeholder changed to a letter but rotated continuously), and a static object (placeholder changed to a letter but never moved). One of the letters was a target (the letter " $\mathrm{S}$ " or "H") on each trial, and the target could appear in any of the four object types.

The other trial type was a conceptual replication of the Sunny and von Mühlenen (2011) method, which will be called New Object trials. In these trials, shown in Fig. 2, three figureeight placeholders were initially displayed on the screen, one of which was moving. At the display transition, the placeholders changed into letters and a new letter appeared. Simultaneously, the initially moving object stopped moving and one of the initially static objects began moving. The display transition resulted in four object types: a motion-onset object (placeholder was initially stationary but the letter began moving at the display transition), a motion-offset object (placeholder was initially moving but stopped moving at the display transition), a static object (placeholder changed to a letter but never moved), and a new object (letter that appeared at the display transition). One of the letters was a target on each trial, and the target could appear in any one of the four object types.

In this experiment and in the subsequent experiment, participants sat in a dimly-lit room, $57 \mathrm{~cm}$ from a computer monitor. Participants' task was to make a key press to indicate whether the target was an "S" or an "H." There was only one target letter on each trial. Participants were told to respond as quickly as possible. The search array remained visible until the participant responded. If the participant responded incorrectly, a brief tone and the message, "Incorrect!" was presented. After each block, participants were informed of their mean reaction time and number of errors.

Design Participants completed 24 practice trials followed by the main experiment, which consisted of eight blocks of 48 trials each. The trial types (New Object and No New Object) were randomly intermixed across the experiment and were equally likely to occur, thus each of the eight object types was a target in 48 trials in the experiment. For each object type, the target letter was equally likely to be "S" or " $\mathrm{H}$," and the distractors were equally likely to be all " $\mathrm{E}$ " or all "U" distractors.

\section{Results and discussion}

Mean reaction times to identify the target are shown in Fig. 3 as a function of the type of object that contained the target, separately for each trial type. ${ }^{1}$ Trials with reaction times 2.5 standard deviations above or below the average reaction time for each target condition for each participant were removed from analysis. For the No New Object trials, there was a main effect of target condition, $F(3,33)=12.23, p<.001, \eta_{p}^{2}=.526$. Follow-up post-hoc comparisons with a Tukey's correction showed that motion onset was significantly faster than motion

\footnotetext{
${ }^{1}$ Data can be found under the Resources tab at http://rabrams.net.
} 


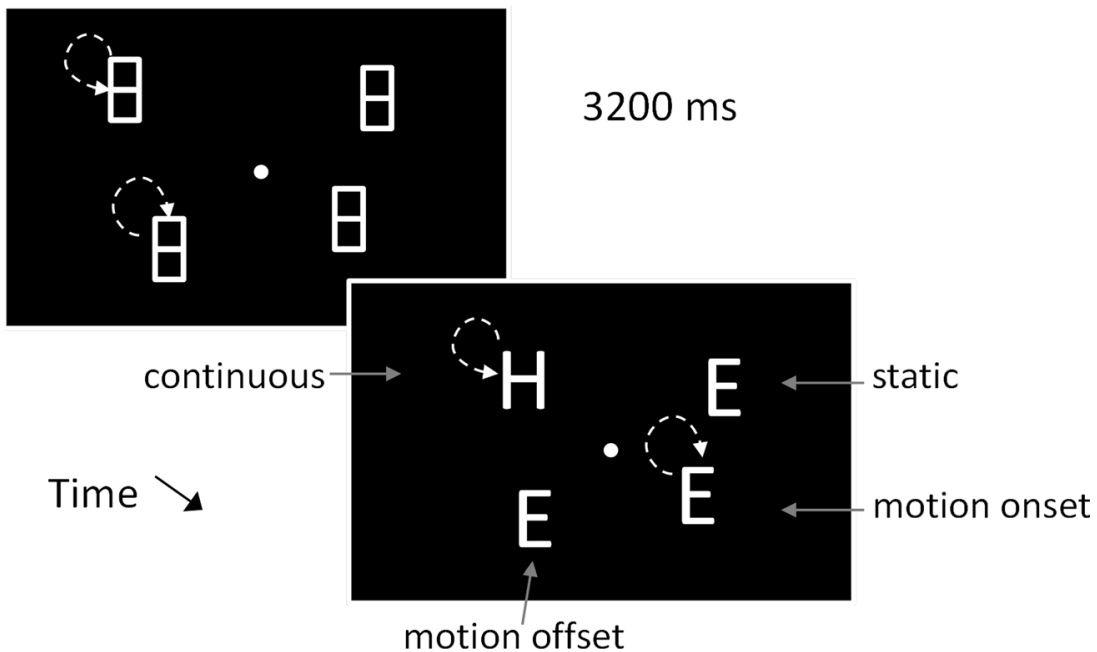

Fig. 1 Sequence of events on a No New Object trial, from Experiment 1. At the start of a trial, two figure-eight placeholders were moving and two were stationary. At the time of the display transition, the figure-eight placeholders changed into letters, one of which was a target.

offset, $t(11)=4.16, p<.001, d=1.69$, motion onset was significantly faster than continuous, $t(11)=5.82, p<.001, d$ $=1.25$, and static was significantly faster than continuous, $t(11)=3.19, p=.02, d=.76$.

For the New Object trials, there was also a main effect of target condition, $F(3,33)=20.74, p<.001, \eta_{p}^{2}=.653$. Followup post-hoc comparisons with a Tukey's correction showed that new object targets were significantly faster than motion onset targets, $t(11)=3.76, p=.004, d=1.58$, motion offset targets, $t(11)=4.68, p<.001, d=2.93$, and static targets, $t(11)=7.82, p$ $<.001, d=2.01$. Motion-onset and motion-offset targets were both significantly faster than static ones $(t(11)=4.06, p=.002, d$ $=.91, t(11)=3.14, p=.02, d=.73$, respectively).
Simultaneously, one of the initially moving objects stopped moving and one of the initially static objects started moving. Curved arrows indicate the motion paths taken by the letters and placeholders, but were not present in the display

Accuracy data are reported in Table 1. For the No New Object trials, there was a main effect of target condition on accuracy, $F(3,33)=6.39, p=.002, \eta_{p}^{2}=.367$. Follow-up posthoc comparisons with a Tukey's correction showed that continuous targets were identified significantly less accurately than onset targets, $t(11)=3.84, p=.003, d=.84$, offset targets, $t(11)=2.79, p=.04, d=.98$, or static targets, $t(11)=3.72, p=$ $.004, d=1.18$. For the New Object trials, there was a main effect of target condition on accuracy, $F(3,33)=3.56, p=.02$, $\eta_{p}^{2}=.245$. Follow-up post-hoc comparisons with a Tukey's correction showed that new object targets were identified significantly more accurately than onset targets, $t(11)=2.91, p=$ $.03, d=1.10$. In both the No New Object and the New Object

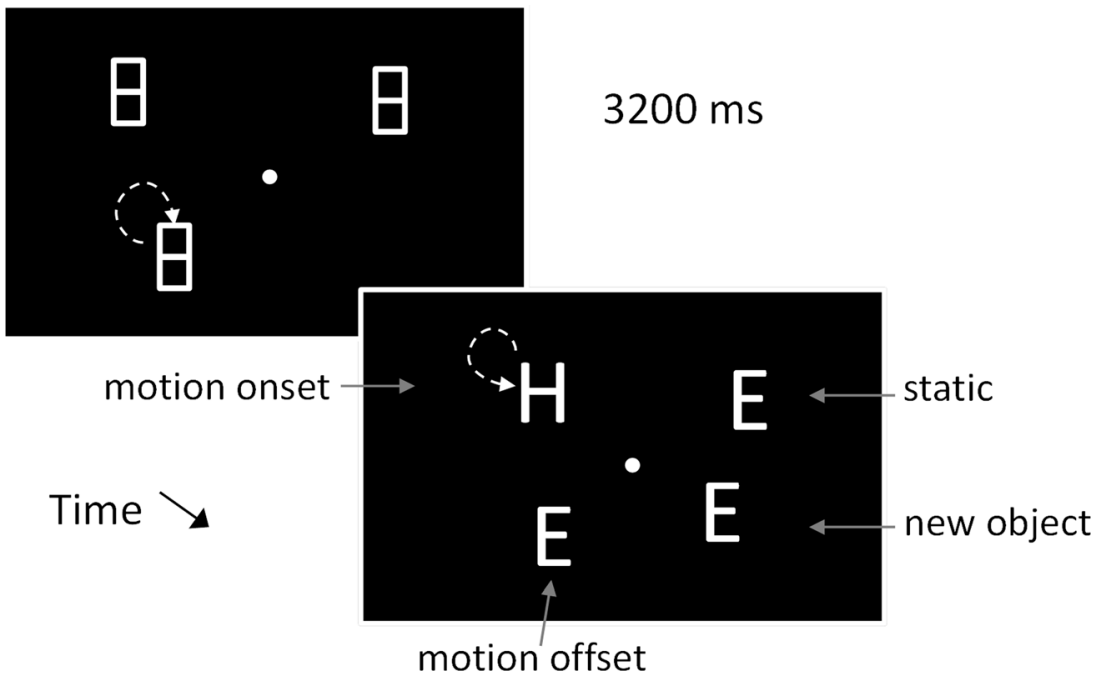

Fig. 2 Sequence of events on a New Object trial, from Experiment 1. At the start of a trial, one of three figure-eight placeholders was moving and two were stationary. At the time of the display transition, the figure-eight placeholders changed into letters. Simultaneously, the initially moving placeholder stopped moving, one of the initially static objects started moving, and a new object appeared. Curved arrows indicate the motion paths taken by the letters and placeholders, but were not present in the display 


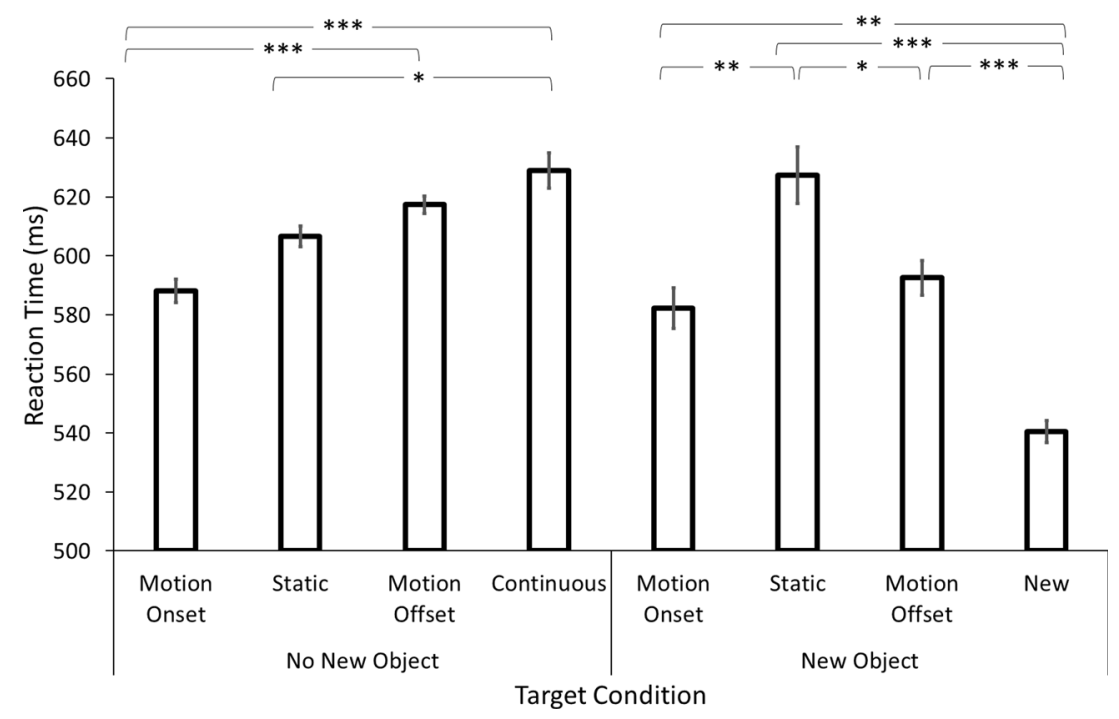

Fig. 3 Results from Experiment 1. Error bars represent within-subject standard error (Cousineau, 2005)

trials, the faster conditions tended to be more accurate, ruling out concerns about speed-accuracy tradeoffs.

Taken together, the results from the present experiment indicate that a new object alters the pattern of attentional capture by animated motion onset. In the No New Object trials, the motion onset target captured attention, as identification of it was significantly faster than for motion offset and continuous targets. However, in the New Object trials, in which a new object abruptly appeared in the display on every trial (as in the experiments of Sunny \& von Mühlenen, 2011), that same stimulus did not capture attention. Thus, the present experiment shows that the inclusion of a new object in the display, as in the experiments by Sunny and von Mühlenen (2011), limits the conclusions that are possible about capture by motion onset.

\section{Experiment 2}

Experiment 2 used analog, natural motion (motion that does not involve animation) to determine if motion onset captures attention. All previous studies examining attentional capture by motion onset used animated motion rather than analog motion. And, as Sunny and von Mühlenen $(2011,2014)$ have shown, the transients inherent to animated displays appear capable of capturing attention; thus, the use of analog motion

Table 1 Proportion correct for each target condition in each trial type

\begin{tabular}{lll}
\hline & No New Object & New Object \\
\hline Motion Onset & .96 & .92 \\
Static & .96 & .95 \\
Motion Offset & .94 & .93 \\
Continuous & .90 & \\
New Object & & .97 \\
\hline
\end{tabular}

in this experiment will help determine if motion onset truly captures attention. If motion onset does capture attention, we expect that the real motion onset used here will capture attention either when it is a feature of the target, a feature of the distractor, or both. The motion was produced here by two physically moving objects that were located adjacent to the search display.

\section{Method}

Participants Twelve participants recruited from the subject pool at Washington University in St. Louis participated for course credit.

Apparatus and procedure As seen in Fig. 4, a black frame holding two motors, each controlling a narrow yellow bar, was mounted to the monitor. The yellow bars were $6^{\circ}$ long

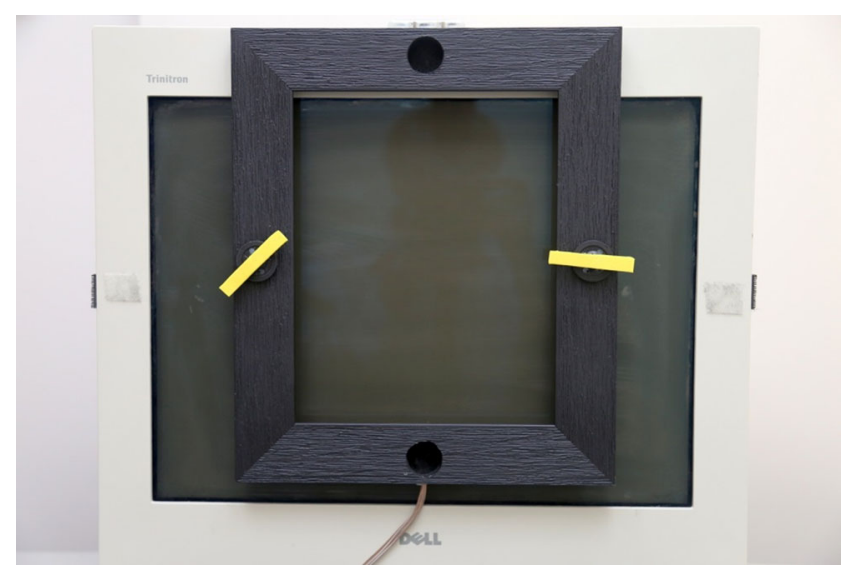

Fig. 4 Apparatus for Experiment 2. Each of two motors could rotate the yellow bars (clockwise) during the trials. The figure-eight placeholders, which changed to letters, appeared on the monitor adjacent to the yellow bars 
and $1^{\circ}$ wide. The sequence of events on a trial is shown in Fig. 5. Two figure-eight placeholders $\left(2^{\circ}\right.$ high and $1^{\circ}$ wide) were displayed $6.2^{\circ}$ to the left and right of fixation, adjacent to the yellow bars which were centered $12^{\circ}$ from fixation. During that time, the yellow bars were each either static or rotating. After 3,200 ms, one or both motors changed their motion, and the placeholders transitioned to letters. The bars rotated at a rate of $538^{\circ} / \mathrm{s}$

The motors were used to produce four motion types: a motion-onset object (yellow bar was stationary but began moving at the transition), a motion-offset object (yellow bar was moving but stopped at the transition), a continuous motion object (yellow bar moved continuously throughout the trial), and a static object (yellow bar never moved).

Participants' task was to indicate via a key press whether the target was an "S" or an " $\mathrm{H}$ ", one of which appeared on each trial. The target letter was always accompanied by an "E" or "U" distractor. Participants were told to respond as quickly as possible. The search array remained visible until the participant responded. If the participant responded incorrectly, a brief tone followed by the message, "Incorrect!" was presented. After each block, participants were informed of their mean reaction time and number of errors.

Design Participants completed 24 practice trials followed by the main experiment, which consisted of eight blocks of 48 trials each. The target was equally likely to appear adjacent to each of the four motion types, and to be an "S" or "H"; the distractor was equally likely to be an "E" or "U" for each motion type. Every combination of target motion and distractor motion was presented, which created sixteen distinct trial types, with 24 trials of each type. The trial types were randomly intermixed across the experiment.

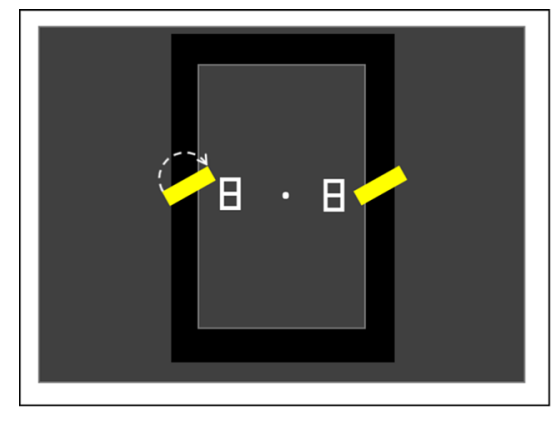

Fig. 5 Sequence of events on a trial in Experiment 2. The two yellow bars, propelled by electric motors, were each initially either rotating or stationary adjacent to two figure-eight placeholders. After 3,200 ms, the placeholders changed into letters, one of which was a target and the other

\section{Results and discussion}

Planned target and distractor analyses Target condition refers to the nature of the motion adjacent to the target letter, and distractor condition refers to the nature of the motion adjacent to the distractor. In this experiment, targets were no more likely to be adjacent to motion onsets, thus any benefit for motion onset as a target or disadvantage for motion onset as a distractor would indicate bottom-up capture by motion onset, given that there is no incentive to direct attention to the motion onset. Trials with reaction times 2.5 standard deviations above or below the average reaction time for each targetdistractor combination for each participant were removed from analysis. A 4 (target condition: onset, offset, static, continuous) $\times 4$ (distractor condition: onset, offset, static, continuous) ANOVA revealed a main effect of target condition, $F(3$, 33) $=5.33, p=.004, \eta_{p}^{2}=.326$. The reaction times for each target condition are shown in Fig. 6. Follow-up post-hoc comparisons with a Tukey's correction on the main effect of target condition showed that motion onset targets were detected significantly faster than motion offset, $t(11)=3.40, p=.009, d=$ .82 , static, $t(11)=3.46, p=.008, d=.86$, and continuous, $t(11)$ $=2.74, p=.046, d=.75$. Importantly, these results indicate that analog motion onset captured attention.

Distractor condition was also analyzed, because an effect of either target or distractor condition could indicate capture by motion onset. The reaction times for each distractor condition are shown in Fig. 7. There was a main effect of distractor condition, $F(3,33)=3.25, p=.03, \eta_{p}^{2}=.228$. Post-hoc comparisons on the main effect of distractor type revealed that static distractors resulted in reaction times for targets that were significantly slower than reaction times for targets in the presence of motion onset distractors, $t(11)=3.10, p=.02, d=.995$.

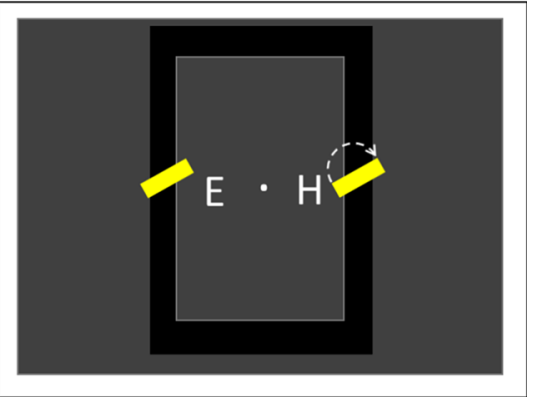

a distractor. Additionally, the motors sometimes produced a motion change, depending on the types of motion they were creating. Arrows indicate the motion paths, but were not present in the display 


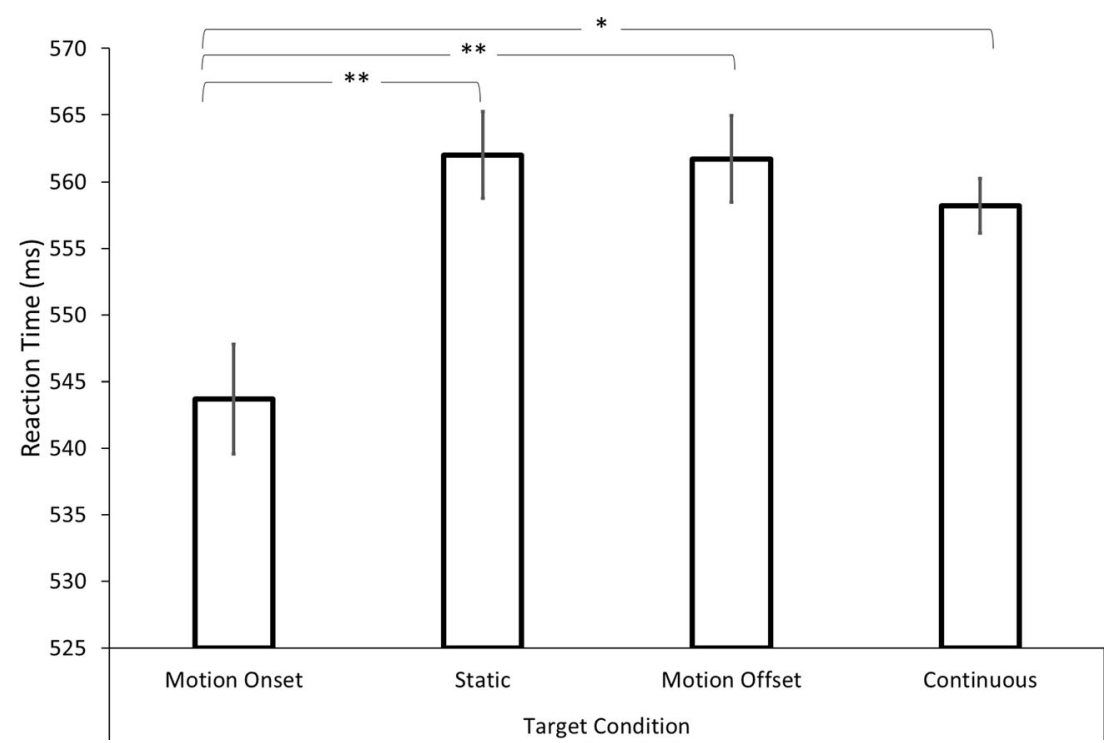

Fig. 6 Reaction times for each target condition from Experiment 2. Error bars represent within-subject standard error (Cousineau, 2005)

There was no significant target condition $\times$ distractor condition interaction, $F(9,99)=1.84, p=.07, \eta_{p}^{2}=.143$.

A 4 (target condition: onset, offset, static, continuous) $\times 4$ (distractor condition: onset, offset, static, continuous) ANOVA on accuracy revealed no significant main effects of target, $F<1$, distractor, $F(3,33)=1.71, p=.184$, or interaction, $F<1$; thus, the reaction time results were not contaminated by a speed-accuracy tradeoff.

The results indicate that motion onset captured attention when adjacent to the target because motion onset targets were identified significantly faster than any of the other types. However, it appears the motion onset did not capture attention when it was adjacent to the distractor because target identification was not slowest when a motion onset object was the distractor. Instead of motion onset capturing attention, it seems possible that motion onset merely produced a general alerting effect when present anywhere in the display, a possibility that we consider next.

Eliminating trials with an identical target and distractor In order to further investigate the possibility that motion onset merely produced a general alerting effect, all trials containing identical target and distractor motion were removed from analysis. The rationale for this is that when both target and distractor are adjacent to the same type of motion, neither one would have an advantage, and such trials do not contribute to our understanding of attentional capture. Importantly, this subset of trials includes those on which motion onset targets were paired with motion onset distractors, potentially reducing any estimate of the magnitude of capture.

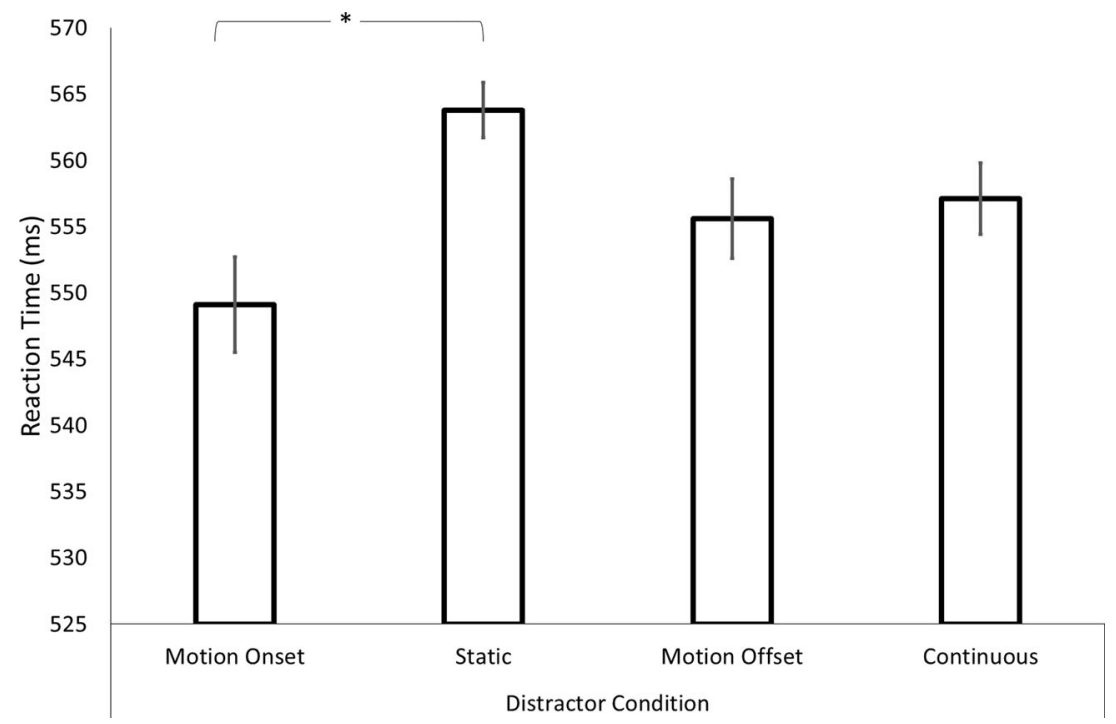

Fig. 7 Reaction time to identify the target as a function of the distractor condition, from Experiment 2. Error bars represent within-subject standard error (Cousineau, 2005) 
As shown in Fig. 8, after removing all of the trials in which the target and distractor condition were identical and collapsing across distractor type, a one-way ANOVA on target condition reaction times revealed a main effect, $F(3,33)=3.83, p$ $=.019, \eta_{p}^{2}=.258$. Follow-up post-hoc comparisons with a Tukey's correction showed that motion onset targets were identified significantly faster than both motion offset targets, $t(11)=3.06, p=.02$, and static ones, $t(11)=2.72, p=.048$, but not continuous, $t(11)=2.31, p=.12$. However, continuous targets were not identified significantly faster than offset, $t(11)$ $=.76, p=.87$, or static, $t(11)=0.41, p=.98$.

A one-way ANOVA on distractor condition in the same subset of trials revealed no main effect, $F(3,33)=1.66, p=.20, \eta_{p}^{2}=$ .13. Thus, the main effect of distractor condition when all trials were considered must have been driven by trials where target and distractor conditions were identical.

In order to confirm that trials in which the target and distractor were identical were responsible for the main effect of distractor when all trials were included in the analysis, a one-way ANOVA was conducted on all trials in which the targets and distractors were identical. The main effect of motion type was significant, $F(3,33)=3.21, p=.036, \eta_{p}^{2}=.226$. Follow-up post-hoc comparisons with a Tukey's correction on the main effect of motion type showed that responses to motion onset $(M=545, S D=34)$ trials were significantly faster than responses to static trials $(M=573, S D=42), t(11)=2.96$, $p=.03$. There was no significant difference in reaction times between the continuous $(M=565, S D=49)$ or offset $(M=$ $566, S D=51)$ conditions and the other conditions. These results indicate that when there were two static objects in the display, participants were significantly slower than when there were two motion onsets in the display. Importantly, the analysis of this subset of trials rules out an alternative interpretation of the main results, and confirms the conclusion that motion onset captured attention.

It may seem that the absence of an effect of distractor type after removing identical target/distractor trials is inconsistent with capture by motion onset. However, because responses were fastest on trials containing two motion onset elements, that suggests that there may also be rapid disengagement from a motion onset element in addition to the rapid engagement shown by capture when the motion onset was near the target. Such rapid disengagement would explain why there was no main effect of distractor condition.

The results from Experiment 2 demonstrate that motion onset captures attention. Importantly, the motion onset in this experiment involved real, analog motion rather than animated motion on a computer display. Therefore, it is not merely the jerkiness of animated motion that produces attentional capture by motion onset, as suggested by Sunny and von Mühlenen $(2011,2014)$, given that natural motion does not have that feature.

\section{General discussion}

The present study shows that natural motion onset does capture attention. When a target letter appeared adjacent to a natural, non-animated motion onset, participants were significantly faster to identify it compared to when the target appeared adjacent to other types of stimuli. Because natural motion onset captured attention (Experiment 2), the effect of attentional capture by motion onset reported in the past is not due solely to the properties of animated motion, as Sunny and von Mühlenen $(2011,2014)$ suggested.

Additionally, Experiment 1 showed that including a new object in a visual search display alters attentional capture by

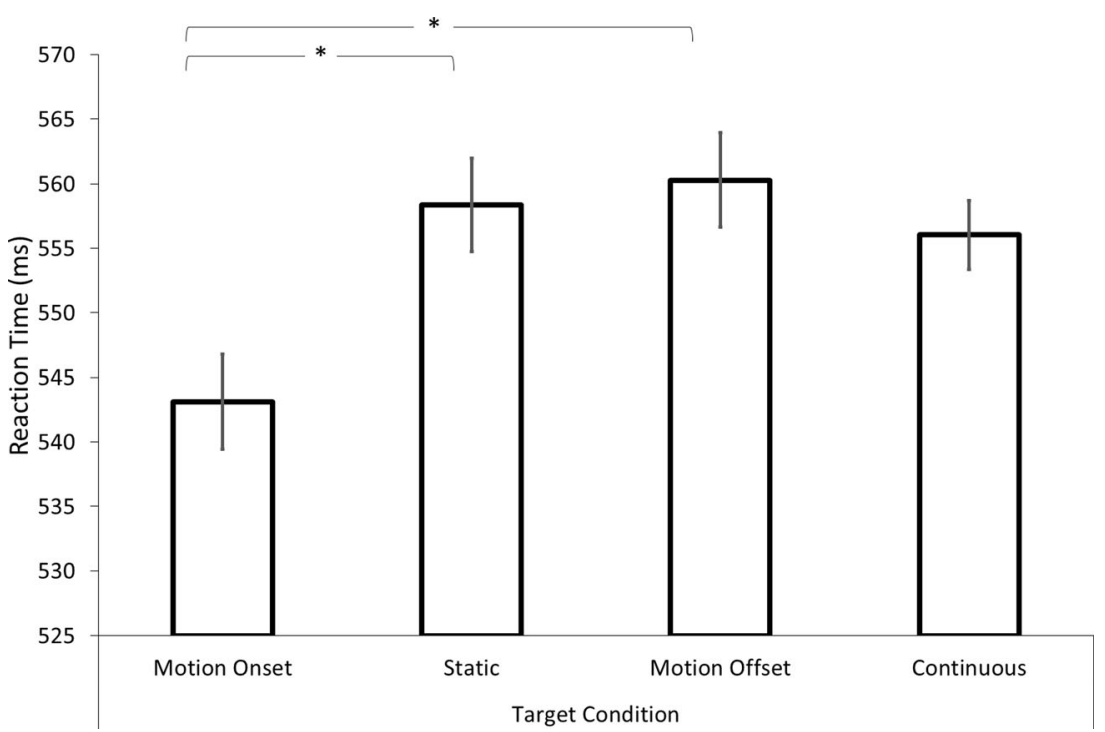

Fig. 8 Reaction times as a function of target condition after removing trials in which target and distractor conditions were identical. Error bars represent within-subject standard error (Cousineau, 2005) 
motion onset; thus, inclusion of a new object in tests of attentional capture by motion onset, as in the experiments by Sunny and von Mühlenen $(2011,2014)$, does not permit an adequate test of whether motion onset captures attention. This conclusion is based on finding an advantage for (animated) motion onset objects in visual search when a new object was not present in the display, but not finding such an advantage when new objects were added to the display (Experiment 1).

\section{Motion onset and new objects}

Experiment 1 indicated that animated motion onset captured attention as it had in the experiments by Abrams and Christ (2003). Additionally, Experiment 1 showed that including a new object in the display suppressed the effect of attentional capture by motion onset: A stimulus that did capture attention under some conditions failed to capture attention in trials in which a new abrupt-onset object was included. That result might have been predicted on the basis of numerous demonstrations that have shown that new objects capture attention (e.g., Christ \& Abrams, 2006; Schrij, Owens, \& Theeuwes, 2008, Yantis \& Johnson, 1990; Yantis \& Jonides, 1984). Additionally, Christ and Abrams (2008) directly examined capture by motion onset in the presence (or absence) of an abruptly appearing new object. They found, as we did here, that new objects reduced attentional capture by motion onset. Given these results, it does not seem surprising that Sunny and von Mühlenen $(2011,2014)$ failed to find capture by motion onset since they included a new object in their display on each trial.

\section{Attentional capture by natural motion}

The present study is the first to examine attentional capture by motion onset using natural motion. All previous studies examining the issue used animated motion (e.g., Abrams \& Christ, 2003; Sunny \& von Mühlenen, 2011, 2014) rendered on a computer display. The animation requires the motion to be simulated by presenting multiple static stimuli in rapid succession. Sunny and von Mühlenen $(2011,2014)$ showed that changes in the animation rate can dramatically alter the attentional effects of the stimuli, revealing a shortcoming of the use of computer displays to address the question.

\section{Limitations and implications}

One potential limitation of the present experiments is that in Experiment 2, the motion occurred adjacent to the placeholders and targets; the search elements themselves did not undergo a motion onset or offset, move continuously, or remain static, as they had in experiments using simulated, animated motion (e.g., Abrams \& Christ, 2003; Sunny \& von Mühlenen, 2011, 2014). However, motion onset captured attention even when it occurred adjacent to the targets, which can be taken as stronger evidence for the claim that motion onset captures attention.

It has been argued that to demonstrate true bottom-up capture, the capture must be insensitive to context and "cognitively impenetrable" (e.g., Folk \& Remington, 2015; Jonides \& Irwin, 1981). In other words, the capture must occur regardless of the other elements or events in the display, and regardless of the observer's goals. Indeed, in Experiment 1, the capture was dependent upon the presence or absence of an onset in the display - apparently violating the criterion of context insensitivity. However, the motion onset used in Experiment 1 was not true analog motion, so conclusions about capture by motion onset are not possible there. Furthermore, because we did not manipulate top-down goals in either experiment, we also cannot conduct a test of the cognitive impenetrability criterion. Thus, it might be most prudent to conclude that we have provided evidence for attentional prioritization caused by motion onset, and not capture according to the stricter definition. Future studies could pursue this issue.

\section{Conclusion}

It has been suggested that motion onsets are prioritized because the onset of motion may reveal the presence of an animal nearby - a potential threat that might require rapid action (Abrams \& Christ, 2003; Pratt, Radulescu, Guo, \& Abrams, 2010). Sunny and von Mühlenen $(2011,2014)$ argued that it was only the slow refresh rate of animated motion onset that captured attention in previous studies, but their experiments included a new object in the display on each trial, which obscured the effect of attentional capture by motion onset. In the present experiments, analog motion onset, without the limitations inherent to animated displays, captured attention. Together, these findings suggest that motion onset really does capture attention.

\section{References}

Abrams, R. A., \& Christ, S. E. (2003). Motion onset captures attention. Psychological Science, 14, 427-432. https://doi.org/10.1111/14679280.01458

Awh, E., Belopolsky, A. V., \& Theeuwes, J. (2012). Top-down versus bottom-up attentional control: A failed theoretical dichotomy. Trends in Cognitive Sciences, 16, 437-443. https://doi.org/10.1016/ j.tics. 2012.06 .010

Christ, S. E., \& Abrams, R. A. (2006). Abrupt onsets cannot be ignored. Psychonomic Bulletin \& Review, 13, 875-880. https://doi.org/10. 3758/BF03194012

Christ, S. E., \& Abrams, R. A. (2008). The attentional influence of new objects and new motion. Journal of Vision, 8, 1-8. https://doi.org/10. $1167 / 8.3 .27$

Cousineau, D. (2005). Confidence intervals in within-subject designs: A simpler solution to Loftus and Masson's method. Tutorials in Quantitative Methods for Psychology, 1, 42-45. 
Enns, J. T., Austen, E. L., Di Lollo, V., Rauschenberger, R., \& Yantis, S. (2001). New objects dominate luminance transients in setting attentional priority. Journal of Experimental Psychology: Human Perception and Performance, 27, 1287-1302. https://doi.org/10. 1037/0096-1523.27.6.1287

Folk, C. L., \& Remington, R. W. (2015). Unexpected abrupt onsets can override a top-down set for color. Journal of Experimental Psychology: Human Perception and Performance, 41, 1153-1165. https://doi.org/10.1037/xhp0000084

James, W. (1890). Attention. In The principles of psychology, Vol I (pp. 402-458). New York, NY, US: Henry Holt and Co. https://doi.org/ 10.1037/10538-011

Jonides, J., \& Irwin, D. E. (1981). Capturing attention. Cognition, 10, 145-150. https://doi.org/10.1016/0010-0277(81)90038-X

Pratt, J., Radulescu, P. V., Guo, R. M., \& Abrams, R. A. (2010). It's alive! Animate motion captures visual attention. Psychological Science, 21, 1724-1730. https://doi.org/10.1177/0956797610387440

Schreij, D., Owens, C., \& Theeuwes, J. (2008). Abrupt onsets capture attention independent of top-down control settings. Perception \& Psychophysics, 70, 208-218. https://doi.org/10.3758/PP.70.2.208
Shioiri, S., \& Cavanagh, P. (1992). Visual persistence of figures defined by relative motion. Vision Research, 32, 943-951. https://doi.org/10. 1016/0042-6989(92)90037-J

Sunny, M. M., \& von Mühlenen, A. (2011). Motion onset does not capture attention when subsequent motion is 'smooth'. Psychonomic Bulletin \& Review, 18, 1050-1056. https://doi.org/10.3758/s13423011-0152-3

Sunny, M. M., \& von Mühlenen, A. (2014). The role of flicker and abrupt displacement in attention capture by motion onsets. Attention, Perception, \& Psychophysics, 76, 508-518. https://doi.org/10. 3758/s13414-013-0587-x

Yantis, S., \& Johnson, D. N. (1990). Mechanisms of attentional priority. Journal of Experimental Psychology: Human Perception and Performance, 16, 812-825. https://doi.org/10.1037/0096-1523.16. 4.812

Yantis, S., \& Jonides, J. (1984). Abrupt visual onsets and selective attention: Evidence from visual search. Journal of Experimental Psychology: Human Perception and Performance, 10, 601-621. https://doi.org/10.1037/0096-1523.10.5.601 